\title{
Ethnicity, Acculturation, and Offending: Findings from a Sample of Hispanic Adolescents
}

\author{
Kristina M. Lopez ${ }^{1}$ and Holly Ventura Miller ${ }^{* 2}$ \\ ${ }^{I}$ Texas State University, USA \\ ${ }^{2}$ University of Texas at San Antonio, USA
}

\begin{abstract}
This study examines the relationship between ethnicity, acculturation, and crime among a sample of Hispanic adolescents drawn from the Project on Human Development in Chicago Neighborhoods (PHDCN) dataset. Prior research has shown that Hispanics who are more acculturated are more likely to engage in crime, but there is a lack of empirical evidence to explain why this is, and little research that has explored Hispanics relative to one another. In an effort to address these shortcomings, this study explores the impact of ethnicity on criminal offending among Hispanic adolescents. This study also examines whether acculturation, net of ethnicity, predicts criminal offending among this group. Using longitudinal data from the PHDCN, we assess the independent effects of ethnicity and generational status, as well as additional criminological variables on adolescent criminal offending. Findings indicated that, on average, Mexican adolescents were less likely than other Hispanics to report violent offending while Puerto Ricans were significantly more likely to report violent offending. No differences were observed between Hispanic subgroups with respect to property offending. Results from negative binomial regression revealed that ethnicity is rendered insignificant in multivariate analyses. Consistent with prior research, first generation immigrants were significantly less likely to engage in delinquent behavior, even after controlling for relevant criminological variables.
\end{abstract}

Keywords: Hispanics, acculturation, immigration, ethnicity and crime.

\section{INTRODUCTION}

The rapid growth of the Hispanic population over the past two decades, along with projected increases in the coming years, will no doubt have considerable impact on social, political, and demographic contexts within the United States [1-4]. Dramatic changes in population demographics, particularly those attributable to immigration, may have implications for research on crime and victimization, especially. Researchers have long considered the link between immigration and crime, most commonly assuming a positive relationship between the two. The dramatic increase in crime during the 1960s and 1970s, for example, coincided with the influx of the post-1965 immigration cohort which, for some, suggested a link between immigration and crime. Early $20^{\text {th }}$ century research also predicted a positive link between immigration and crime $[5,6]$, but recent research by Martinez and colleagues has challenged this notion, suggesting immigrants' involvement in crime may be lower than predicted [7-9].

The relationship between immigration and crime has been examined within both micro and macro-level analyses. As such, the research questions vary depending on the level of analysis. For macro analyses, the question relates to whether immigration leads to variation in crime rates, while micro-level analyses examine whether immigrants are more likely to be involved in crime relative to the native born. This study addresses the latter, whether first-generation

*Address correspondence to this author at the University of Texas at San Antonio, USA; Tel: 201-458-2688; Fax: 201-458-2680;

E-mail: holly.miller@utsa.edu immigrants are more or less likely to offend compared to their second and third-generation counterparts.

The link between immigration and crime at the individual level has often been explored through the framework of the acculturation process. Acculturation is defined as the interaction of two distinct cultures, resulting in a minority group's adoption of a host society's values, beliefs, and behavior [10]. Researchers have debated the nature of acculturation process for immigrants and how it impacts their criminal involvement. Park and Burgess, for example, favored classical assimilation theory which describes a linear process toward more positive socioeconomic outcomes as immigrants assimilate into the American mainstream [5]. Conversely, Portes and Zhou [11] proposed an alternate theory of segmented assimilation, wherein immigrants may not assimilate into the American mainstream, but instead either 1) maintain their own culture to advance further socioeconomically, or 2) assimilate into the urban underclass of the native poor. Segmented assimilation attempts to explain how post-1965 immigrants may assimilate relative to, and perhaps in contrast with, late $19^{\text {th }}$ and early $20^{\text {th }}$ century immigrants. Some researchers have argued that post1965 immigrants may not assimilate in a fashion similar to previous cohorts because of the different demographic makeup of new immigrants and the dissimilar economy that now exists [11-14].

While there is a considerable body of research related to immigration and crime (see Martinez and his colleagues, for example [7-9]), there is an absence of literature that examines the specific ethnicities which comprise immigrant populations. In particular, the relationship between 
assimilation and crime among the varying ethnicities comprising the Hispanic population has yet to be examined [8]. Given the rapid growth of the Hispanic population, a better understanding of these diverse groups is important to disentangling the effects of ethnicity and immigration on crime.

The current study is designed to add to the literature related to immigration, ethnicity, and crime in two important ways. First, the study examines the differences in criminal offending among three Hispanic subgroups, Mexicans, Puerto Ricans, and other Hispanics (e.g. those from Spain, Central American, South American, and other Hispanic ethnicities) and assesses the role of ethnicity in the etiology of offending. Second, we analyze the link between acculturation, as measured by generational status, and offending behavior among these Hispanic adolescents. Though generational status is not a perfect measure for acculturation levels, it has often been used as a proxy in previous studies [14-16] and is the most appropriate variable available in the PHDCN. Using a series of negative binomial regression models, we estimate the effects of ethnicity, acculturation, and theoretically relevant control variables on property, violent, and overall self-reported delinquency among Hispanic adolescents.

\section{BACKGROUND}

\section{Acculturation, Ethnicity, and Crime}

The relationship between immigration and crime has been explored by historians, sociologists, and criminologists for decades. Early research hypothesized a positive link between the presence of immigrants and crime such that crime rates would be higher in areas of greater immigrant concentration, however, evidence failed to confirm this hypothesis [6]. Today, there is even less empirical research supporting a connection between immigration and crime [79], yet there remains a tendency to identify immigrants as responsible for a disproportionate amount of crime [14].

There has been, however, a large body of research suggesting that the children and grandchildren of immigrants (i.e., the second and third generation) are far more likely to experience negative outcomes, including behavioral problems. Sociologists have argued that high rates of crime among second generation immigrants are due to a departure from their own cultural traditions and assimilation into the American mainstream [17-19]. According to this perspective, acculturation weakens the impact of social controls in immigrant communities by creating a conflict between the cultural values of immigrants and the legal codes constructed by native groups [7]. Empirically, the process of acculturation has been shown to have positive and negative results on both immigrants and neighborhoods. Newly arrived immigrants may negatively disrupt a neighborhood by weakening social bonds and community cohesion; conversely, the neighborhood may have a negative impact on the immigrants, exposing them to the delinquent subcultures of the native poor [9].

In addition to acculturation status, immigrants' nation of origin is essential to studying the progression across generations. A fundamental shortcoming of the majority of research conducted on Hispanics is the lack of differentiation of national origin. Since Hispanics are not a monolith, instead encompassing dozens of distinct groups, studies that fail to acknowledge and control for these differences run the risk of erroneous findings and faulty implications for Hispanic populations in terms of policy and practice. The differences between these groups are much more than mere country of origin, however. In fact, significant differences exist on a number of social and economic indicators that may have particular import for the study of Hispanic crime and victimization.

The few studies which have controlled for ethnicity or nation of origin have indicated some variability among these groups across a number of social, economic, and behavioral outcomes $[8,16,20]$. Data from the American Community Survey and the U.S. Census [21] also reveal many social and cultural differences between various Hispanic/Latino groups that may correlate with variability in criminal involvement and victimization, including age, marital status, fertility, household type, employment, and education. For example, while approximately one-quarter of the overall U.S. population is under the age of 18 , more than a third of all Hispanics $(34.3 \%)$ are younger than 18 . This number varies by subgroup (Mexicans $=36.6 \%$; Puerto Ricans $=33.7 \%$; Cubans $=21.2 \%)[21]$.

Hispanics are about as likely as the rest of the population to be married and less likely to have divorced (10.2\% vs $7.5 \%)$. Again, numbers vary by sub-group with Cubans and Puerto Ricans (12.3\% and $11.4 \%$, respectively) more likely than Mexicans (6.2\%) to be divorced. Mexicans report much higher birthrates compared to other Hispanic sub-groups (Mexican=82.8 per 1,000; Puerto Rican=69.7 per 1,000; Cuban=41.7 per 1,000) resulting in larger families [21].

While all Hispanic groups have higher incidence of single-female headed households than the general U.S. population, this too varies by sub-group. More than onequarter of all Puerto Rican households are headed by females $(26.6 \%)$; this number is significantly lower for both Mexicans (17.3\%) and Cubans (14.6\%). Educational and employment also vary by place or origin, with only half of all Mexicans graduating from high school (Puerto Rican $=71.4 \%$; Cuban $=74.2 \%$ ) and only $17.7 \%$ of this group employed in management, professional and related occupations (Puerto Rican=25.7\%; Cuban=30.9\%). Hispanics, overall, make significantly less than the median household income for the population as whole $(\$ 35,929$ vs $\$ 44,684)$, and this too varies by sub-group (Mexican $=\$ 35,185 ;$ Puerto Rican $=\$ 34,092 ;$ Cuban $=\$ 38,256$ ) [21].

Each of these has the potential to impact and confound our understanding of Hispanic crime and victimization, and may have particular import for policy and practice formulation. Because of variation in the correlates of crime among these diverse groups, it is sensible to explore whether differences exist in criminal offending as well.

\section{Classical \& Segmented Assimilation Theories}

The assimilation of immigrants into American society has long been of great interest to sociologists and historians. Two main perspectives describe the experiences of immigrants in the United States, classical assimilation and segmented assimilation. Classical assimilation theory 
contends that once assimilation has begun, it will proceed in a "straight-line" fashion, with a steady progression toward more positive socioeconomic outcomes across succeeding generations [14]. This theory emphasized the intimate and intense interaction by ethnic minorities with the "primary group" of a host society necessary to assimilate through common neighborhoods and schools. Milton Gordon [10] extended this theory by suggesting "structural assimilation" is the key to achieving full assimilation by ethnic minorities, building relationships with the primary group in order to ensure ethnic incorporation into the mainstream.

Over time, classical assimilation theory came under criticism for the lack of attention to the contextual realities of today's immigrant populations. Portes and Zhou [11] introduced segmented assimilation which attempts to explain how new immigrant groups will assimilate in the face of dramatically different circumstances. This perspective has two main differences from classical assimilation theory. First, the theory hypothesizes that immigrant youth who assimilate into disadvantaged neighborhoods without strong family ties and community support from co-ethnics have a high probability of adopting inner-city youth subcultures, which place less emphasis on education and offer greater encouragement for criminal behavior [22]. Second, this perspective acknowledges the possibility that immigrant groups may reject assimilation into the American mainstream and instead retain their cultural characteristics through the ethnic enclave. While classical assimilation predicts that immigrants who assimilated into the primary group will experience socioeconomic advancement, segmented assimilation predicts negative outcomes for immigrants acculturating into native-born subcultural realities.

Segmented assimilation focuses on various factors such as the timing of an immigrant group's arrival, government policies, the extent of ethnic group discrimination, and the community structure into which they immigrate [11]. Segmented assimilation suggests that as immigrant generations progress, they become more exposed to features of deleterious inner city culture. As a result, they may become more likely to adopt values tolerant of, or conducive to, criminal offending. Thus, second and third generation immigrants will be more likely to engage in crime and delinquency as compared to their first generation counterparts.

In one of the few tests to date of the segmented assimilation hypothesis, Morenoff and Astor [14] analyzed data based on self-reported violent offending among adolescents living in Chicago neighborhoods and came to four conclusions. First, their findings suggested that immigrants face a negative downward assimilation, becoming more involved in crime as they acculturate into American society. Second, results showed immigrants with longer U.S. residency had higher odds of various types of violent behavior. Third, the authors' results indicated that the relationship between age of arrival into the U.S. and crime was not as strong as the relationship between acculturation and involvement in violence. Finally, data revealed a link between neighborhood context and violence. Specifically, third generation youth residing in disadvantaged neighborhoods posed the highest risk of violent behavior.
Overall, these findings generally supported the segmented assimilation hypothesis.

Other research has suggested that criminal offending is relatively low among first-generation immigrants but higher among the descendants of these immigrants. With results varying by city, most researchers have concluded that first generation immigrants are less prone to criminal conduct [23]. Results typically indicate that the children and grandchildren of immigrants have higher crime rates than their parents, but also have lower crime rates than nativeborn children [7]. Morenoff and Astor's study [14], although the first to consider the role of acculturation on violent behavior, did not focus explicitly on the Hispanic subsample of the PHDCN, instead used data from all immigrants in the sample. This study, conversely, will focus on the Hispanic adolescents and thus be able to shed light on the specific experiences of this rapidly growing demographic. Furthermore, this study will be the only to date which controls for ethnicity such that any differences between these groups will be elucidated.

Beyond the Morenoff and Astor study [14], there is good reason to believe that the process of acculturation may be linked to criminal offending. Various social, health and behavioral outcomes have been explored relative to the acculturation process and most of the studies have shown that higher levels of acculturation are linked to a greater incidence of negative outcomes overall [24-29]. Research has documented numerous psychological and health outcomes such as depression, psychological distress, poor nutrition, and prenatal health behaviors as linked to Hispanics' acculturation process [30-33]. Collectively, these studies indicate that the foreign born (i.e., first generation) experience significantly less health disorders than the native born $[26,29,34]$.

Generational status among Hispanics has also been associated with negative behavioral conduct such as abuse of alcohol and drug and non-enrollment in school [16, 27, 3539]. A considerable body of research has indicated that native-born Hispanics are more likely to use and abuse alcohol, marijuana, inhalant, cocaine and cigarettes [16, 27, 35, 36, 38-40]. Recent research by Maldonado-Molina and her colleagues $[41,42]$ indicate that first generation Hispanic immigrants are significantly less likely to report alcohol and marijuana use as well as report driving under the influence. Important for the current study, their research also suggested that first generation Hispanics are at less risk for physical aggression as well.

\section{The Current Study}

The current study is intended to extend the extant literature by examining the relative effects of ethnicity and acculturation, net of control variables, on crime. More specifically, this study is designed to examine two research questions. First, this research explores if criminal offending varies across Hispanic subgroups (i.e., Mexican, Puerto Rican, and other Hispanics). Unfortunately, there is an absence of literature examining the experiences of these Hispanic sub-groups and it has yet to be established if participation in crime and delinquency varies by ethnicity. Second, this research also examines if generational status, net of ethnicity and other control variables, impacts criminal 
offending among Hispanic adolescents. Based on past research, we hypothesize that second and third generation Hispanic youths will be more likely to engage in criminal behavior compared to those more newly arrived immigrants (i.e. first generation). The hypothesis challenges classical assimilation theory, which predicts those immigrants whom assimilate into American culture are less likely to be involved in criminal behavior. Conversely, our hypothesis is consistent with the theory of segmented assimilation which predicts an increased involvement in criminal behavior for the second and third generation.

\section{METHODOLOGY}

\section{Data}

The data used for the current analysis are from the Project on Human Development in Chicago Neighborhoods (PHDCN), a longitudinal study of how neighborhoods impact the behavior and psychological development of children and adolescents. These data are appropriate for assessing the impact of both ethnicity and generational status on the probability of delinquent behavior among Hispanic adolescents due to the oversampling of Hispanics in the original research design. Approximately 45 percent of this sample consists of Hispanic children and adolescents with varying generational statuses.

Aimed at creating a large, randomly selected dataset, the PHDCN is composed of two sampling components: the selection of neighborhood clusters and the selection of dwellings. First, utilizing stratification sampling on seven groupings of racial/ethnic composition and three levels of socioeconomic status, 847 census tracts were selected and delineated into 343 neighborhood clusters (NCs) [43-46]. The NCs averaged approximately 8,000 people each and were based on a combination of geographic boundaries and knowledge of Chicago neighborhoods. In effort to observe the physical, social, and economic characteristics within the 343 NCs, a stratified probability sample of 80 NCs was taken [43].

Following the selection of the $80 \mathrm{NCs}$, random selections of block groups were taken from each of these and collapsed into approximately 40,000 dwelling units through inscreening. Infants, children, and adolescents (including 18 year olds) were observed over a seven year period to study their development from infancy and early to mid adulthood relative to influences from community and family. Participants were within approximately six months of the following age cohorts: $0,3,6,9,12,15$, and 18 years. The longitudinal study's data collection took place in three waves: 1994-1997, 1997-1999, and 2000-2001, allowing an approximate 2.5 year gap between waves.

\section{Analysis Sample}

The analysis sample was taken from waves 1 and 2 cohorts of 9,12 and 15 year old children, adolescents and their primary caregivers, whom were self-identified as Hispanic. Specifically, all independent variables were drawn from wave 1, while the dependent measures are drawn from wave 2. The analysis sample was a total of 763 Hispanic children consisting of $71 \%$ Mexican, $18 \%$ Puerto Rican, and $11 \%$ 'other' Hispanic. The $11 \%$ 'other' Hispanic consists of representation from Spain, Central American, South
American, and other Hispanic ethnicities. The analysis sample consists of $51 \%$ males. These children and their families resided across 59 of the 80 neighborhood clusters that were randomly selected for the longitudinal study.

\section{Variables}

\section{Dependent Variables}

Self-Report Offending. The occurrence of criminal behavior was measured using a Self-Report Offending (SRO) instrument [47]. Specifically, subjects were asked questions to self-report their property, violent, and drug offending in a 12 month period before the wave 2 interviews. The questions for each subject consisted of how many times he/she committed that offense. Thus, the offending measures are count variables. Twenty different offenses were addressed in the questions to measure self-reported offending: thirteen violent offenses (e.g., shot someone; been in a gang fight; attacked someone with a weapon) and seven property offenses (e.g., purposely damaged or destroyed property not belonging to you; stolen something from a store; and entered or broken into a building to steal something). For this analysis, the total offending measure also was broken up into two distinct scales, one denoting violent offending and one denoting property offending. The response to the questions are summed so that higher values denote higher levels of offending. A third scale measuring the total number of offenses was also created where higher values indicate greater involvement in delinquency. Specific items for each outcome measure can be found in the Appendix.

\section{Independent Variables}

Ethnicity. Three variables were used to denote ethnicity. These measures were dichotomous variables that indicate if the respondent is Mexican, Puerto Rican, or other Hispanic (comprised of individuals from Central American, South American, Spain, and other Hispanics) ${ }^{1}$.

Acculturation (Generational) Status. Three variables were used to measure generational status. Subjects coded as first generation were those children and adolescents whom were born outside of the United States. Those coded as second generation were born in the U.S. but had at least one parent that migrated from outside of the U.S. Children and adolescent subjects whose parents and themselves were born in the U.S., but had at least one grandparent born outside the U.S. were coded as third generation. Generational status was measured at Wave 1 and is dummy coded.

Self-Control. Previous research has established that models not accounting for self-control risk specification errors [48]. Thus, a measure of self-control was included as a control variable in this analysis. The 17 behavioral items from the EASI-temperament instrument (see the Appendix for all measures) is used to measure self-control in this study [49]. At wave 1, primary caregivers were asked to report on their children answering multiple questions, focusing on the child's inhibitory control, decision time, sensation seeking, and persistence. Subjects were asked questions including:

\footnotetext{
${ }^{1}$ Due to small numbers of these other Hispanic subgroups, this third group was collapsed to provide meaningful comparisons between the two largest Hispanic groups, Mexicans and Puerto Ricans, and all other Hispanics.
} 
five inhibitory control (e.g., has trouble controlling his/her impulses), four decision time (e.g., often acts on the spur of the moment), four sensation seeking (e.g., tends to get bored easily), and four persistence (e.g., tends to give up easily).

Each participant's item responses are summed and averaged across the 17 behavioral items. Scores were coded so that higher scores on the scale indicate lower levels of self-control (i.e., low self-control) $(\alpha=.68)$. Previous studies that utilized the PHDCN validated this measure [49].

Delinquent Peers. As with self-control, models that fail to account for delinquent peers may risk specification error [50]. To measure peer delinquency, 20 items from the Deviance of Peers instrument were administered to children and adolescents in wave 1 (see Appendix). This self-report interview collected information regarding minor and serious delinquency between peers in the past 12 months [47]. Participants were asked how many of their peers engaged in trivial forms of delinquency, property, and violent crimes. The responses to the questions were scaled as 1 (none of them) to 3 (all of them). Questions were posed such as: "in the past year how many of your friends you spend time with have done the following things: skipped school; stolen something worth $\$ 5$ but less than $\$ 500$; hit someone with the idea of hurting them; sold drugs, such as heroin, cocaine, or crack?" Responses to items were summed with higher scores indicate a greater number of delinquent peers $(\alpha=.87)$.

Demographic Characteristics. Utilizing household income, maximum education level of primary caregiver and partner, and the socioeconomic index (SEI) for primary caregiver's and partner's jobs, socioeconomic status is measured with the combination of these three factors ${ }^{2}$. Values were then standardized with a mean of 0 and a standard deviation of 1 . Positive scores indicate SES higher than the sample average and negative scores indicate SES lower than the sample average. The subjects' gender is coded 1 (male) or 0 (female). The participant's age at wave 1 is measured as a continuous variable.

\section{Analytic Strategy}

This study utilizes a variety of statistical techniques to examine the specified research questions. First, descriptive statistics were used to provide an overview of the sample including measures of central tendency and dispersion. Second, correlations were calculated to assess any bivariate relationships between our variables of interest. Next, in order to assess variation in offending behavior across ethnicity, independent samples t-tests were employed. T-tests are used to explore differences in means, in this case, the t-test was used to explore if the average level of offending varies by ethnicity (i.e., Mexican vs Puerto Rican, Mexican vs other Hispanic, etc).

In order to examine the relative effects of the independent variables on the outcome measure of criminal offending, this study employed negative binomial regression. Negative binomial regression models information on counts, particularly when there is no naturally observed upper limit bounding these counts, which prevents the use of techniques

\footnotetext{
${ }^{2}$ The SES measure was created by the original data collection team and was included in the public use version. Thus, this study did not create the measure but used the previously created and validated measure.
}

based on observed proportions. This analysis proceeds by estimating a series of models designed to assess the effects of ethnicity, acculturation, and germane control variables on property, violent, and overall offending. Models 1-3 regresses self-reported property offending on ethnicity, acculturation, and the controls in a stepwise manner. Model 1 includes only ethnicity variables and demographics, model 2 adds acculturation to the equation, and model 3 regresses the property offending scale on all of the independent variables. This process was repeated for the two other outcomes, violent offending (models 4-6) and overall offending (models 7-9). By estimating these models in a stepwise fashion, we are better able to assess if the relationships between ethnicity and crime and acculturation and crime can be explained through the utilization of relevant criminological controls variables such as demographics, delinquent peers, and self-control.

\section{FINDINGS}

Table 1 presents the sample's descriptive statistics. The sample is comprised of 763 Hispanic adolescents ranging between the ages of 7 and 15 (Mean $=11.92$ years, S.D. $=2.41$ ). The sample used for the analysis is $51 \%$ male, $71 \%$ Mexican, $18 \%$ Puerto Rican, and 11\% other Hispanic ethnicity. The sample contains $24.2 \%$ first generation, $70.1 \%$ second generation, and $5.7 \%$ third or fourth generation Hispanic immigrants. Table $\mathbf{1}$ also reports delinquent peer associations which range from 20 to 51 (Mean=28.42, S.D. $=5.59$ ) with higher scores reflecting a greater number of delinquent peers. The self-control measure had a minimum score of 17 and maximum score of 76 (Mean=42.95, S.D. $=11.32$ ) with higher scores indicating less self-control. The measure denoting socioeconomic status ranged from 2.99 to 3.11 with a mean score of $-.74($ S.D. $=1.17)$. Higher scores on the socioeconomic measure reflect greater socioeconomic status (i.e., higher household income, maximum education level of primary caregiver and partner, and the socioeconomic index (SEI) for primary caregiver's and partner's job). Since the mean is slightly less than 0 , the analysis sample $(n=763)$ has a lower average SES than the total PHDCN sample.

The total self-report offending measure combines twenty different offenses: thirteen violent offenses (e.g., carried a weapon; shot someone, been in a gang fight; attacked someone with a weapon) and seven property offenses (e.g., purposely damaged or destroyed property not belonging to you; stolen something from a store; and entered or broken into a building to steal something). The self-report total offending scale ranged from 0 to 12 with an average of .71 and a standard deviation of 1.46 . Of the thirteen violent offenses, the sample reported committing a minimum of 0 and maximum of 6 violent offenses (Mean=.41, S.D. $=.96$ ). Of the seven property offenses, subjects reported a minimum of 0 and maximum of 6 property offenses (Mean=.25, S.D. $=.62$ ).

Table 2 presents the correlation matrix. Correlations were calculated in order to assess the bivariate relationships between our variables of interest. Only significant correlations are discussed. Mexican youth were significantly less likely to report violent behavior, as compared to their non-Mexican counterparts $\quad(\mathrm{r}=-.13, \mathrm{p}<.05)$. Similarly, 
Table 1. Descriptive Statistics for PHDCN Variables $(n=763)$

\begin{tabular}{|c|c|c|c|c|c|}
\hline \multicolumn{2}{|c|}{ Variables } & Mean & SD & Minimum & Maximum \\
\hline \multicolumn{2}{|c|}{ Self-Report Violent Offending } & .41 & .96 & 0 & 6 \\
\hline \multicolumn{2}{|c|}{ Self-Report Property Offending } & .25 & .62 & 0 & 6 \\
\hline \multicolumn{2}{|c|}{ Self-Report Total Offending } & .71 & 1.46 & 0 & 12 \\
\hline \multicolumn{2}{|l|}{ Age } & 11.92 & 2.42 & 7.77 & 15.69 \\
\hline \multicolumn{2}{|l|}{ SES } & .7410 & -1.18 & -3.16 & 2.93 \\
\hline \multicolumn{2}{|l|}{ Delinquent Peers } & 28.42 & 5.60 & 20 & 51 \\
\hline \multicolumn{2}{|l|}{ Self-Control } & 42.95 & 11.32 & 17 & 76 \\
\hline \multicolumn{6}{|l|}{ Percentages } \\
\hline \multicolumn{6}{|c|}{ Gender $(1=$ Male $) \quad 51.0 \%$} \\
\hline \multicolumn{6}{|c|}{$1^{\text {st }}$ Generation $\quad 24.2 \%$} \\
\hline \multicolumn{6}{|c|}{$2^{\text {nd }}$ Generation $\quad 70.1 \%$} \\
\hline \multicolumn{6}{|l|}{$3^{\text {rd }}$ Generation } \\
\hline \multicolumn{6}{|l|}{ Mexican $\quad 71 \%$} \\
\hline Puerto Rican & $18 \%$ & & & & \\
\hline Other Hispanic & $11 \%$ & & & & \\
\hline
\end{tabular}

Mexican ethnicity was significantly correlated with the total self-report offending scale $(\mathrm{r}=-.12, \mathrm{p}<.05)$. Mexicans were significantly more likely to be first generation immigrants $(\mathrm{r}=.10, \mathrm{p}<.05)$ and less likely to be third generation immigrants $(\mathrm{r}=-.20, \mathrm{p}<.05)$. Mexican ethnicity was also negatively correlated with age $(\mathrm{r}=-.08, \mathrm{p}<.05)$, SES $(\mathrm{r}=-.30$, $\mathrm{p}<.05)$, delinquent peers $(\mathrm{r}=-.15, \mathrm{p}<.05)$, and low self-control $(\mathrm{r}=-.14, \mathrm{p}<.05)$.

Puerto Rican youth were more likely to report violent offending $(\mathrm{r}=.10, \mathrm{p}<.05)$ than other Hispanics, and PR ethnicity was significantly correlated with the total selfreport offending scale $(\mathrm{r}=.08, \mathrm{p}<.05)$. Puerto Rican respondents were less likely to be first generation immigrants $(r=-10, p<.05)$ and more likely to be third generation $(\mathrm{r}=.30, \mathrm{p}<.05)$. Puerto Rican ethnicity also positively correlated with SES $(\mathrm{r}=.21, \mathrm{p}<.05)$, delinquent peers $(\mathrm{r}=.10, \mathrm{p}<.05)$, and low self-control $(\mathrm{r}=.15, \mathrm{p}<.05)$. Fewer significant correlations were found between the "other Hispanic" variable and the other measures. Other Hispanic groups were less likely to be third generation immigrants $(\mathrm{r}=-.08, \mathrm{p}<.05)$ and more likely to associate with delinquent peers $(\mathrm{r}=.10, \mathrm{p}<.05)$. The only other significant relationship was with SES $(\mathrm{r}=.12, \mathrm{p}<.05)$. No significant bivariate relationships emerged between other Hispanic ethnicity and any of the offending measures.

Finally, first generation immigrant status was weakly, but significantly, related to age $(\mathrm{r}=.08, \mathrm{p}<.05)$, SES $(\mathrm{r}=-.21$, $\mathrm{p}<.05)$, low self-control $(\mathrm{r}=-.10, \quad \mathrm{p}<.05)$, and violent

Table 2. Correlation Matrix

\begin{tabular}{|c|c|c|c|c|c|c|c|c|c|c|c|c|c|c|}
\hline Variable & $\mathrm{X} 1$ & $\mathbf{X} 2$ & $\mathbf{X 3}$ & $\mathrm{X} 4$ & X5 & X6 & $\mathrm{X} 7$ & $\mathrm{X8}$ & $\mathbf{X 9}$ & $\mathbf{X 1 0}$ & $\mathbf{X 1 1}$ & Y1 & Y2 & Y3 \\
\hline Sex & 1.0 & & & & & & & & & & & & & \\
\hline SES & .03 & .01 & 1.0 & & & & & & & & & & & \\
\hline Mexican & -.06 & $-.10^{*}$ & $-.30 *$ & 1.0 & & & & & & & & & & \\
\hline Other Hispanic & -.01 & .04 & $.12 *$ & $-.50^{*}$ & $-.15^{*}$ & 1.0 & & & & & & & & \\
\hline $1^{\text {st }}$ Generation & .05 & $.10^{*}$ & $-.21 *$ & $.10 *$ & $-.10^{*}$ & -.01 & 1.0 & & & & & & & \\
\hline $2^{\text {nd }}$ Generation & -.07 & $-.10^{*}$ & $.11 *$ & .04 & -.05 & .05 & $-.87 *$ & 1.0 & & & & & & \\
\hline $3^{\text {rd }}$ Generation & .05 & .03 & $.16^{*}$ & $-.20^{*}$ & $.29 *$ & $-.08 *$ & $-.13^{*}$ & $-.37 *$ & 1.0 & & & & & \\
\hline Violent Offending & $.13^{*}$ & $.22 *$ & $.10 *$ & $-.13^{*}$ & $.10^{*}$ & .06 & $-.09^{*}$ & .04 & $.10^{*}$ & $.34 *$ & $.12 *$ & $.42 *$ & 1.0 & \\
\hline Total Offending & $.11^{*}$ & $.21^{*}$ & $.10 *$ & $-.12 *$ & $.10^{*}$ & .06 & -.07 & .03 & $.08 *$ & $.34 *$ & $.11^{*}$ & $.74 *$ & $.91^{*}$ & 1.0 \\
\hline$* p<.05$ & & & & & & & & & & & & & & \\
\hline
\end{tabular}


Table 3. Individual Sample T-Tests, Differences of Means

\begin{tabular}{|c|c|c|c|c|c|c|c|c|c|}
\hline & \multicolumn{3}{|c|}{ Mexican vs non-Mexican } & \multicolumn{2}{c|}{ Puerto Rican vs non-Puerto Rican } & \multicolumn{3}{c|}{ Other Hispanic vs Mexican/Puerto Rican } \\
\cline { 2 - 9 } & Mean (S.D.) & Mean (S.D.) & t-Statistic & Mean (S.D.) & Mean (S.D.) & t-statistic & Mean (S.D.) & Mean (S.D.) & t-Statistic \\
\hline \hline $\begin{array}{c}\text { Violent } \\
\text { Offending }\end{array}$ & $.33(.82)$ & $.60(1.20)$ & $3.522^{* *}$ & $.59(1.25)$ & $.37(.88)$ & $-2.418^{*}$ & $.58(1.18)$ & $.38(.93)$ & -1.529 \\
\hline $\begin{array}{c}\text { Property } \\
\text { Offending }\end{array}$ & .23 & .30 & 1.449 & .31 & .23 & -1.206 & .25 & .24 & -.077 \\
\hline
\end{tabular}

offending $(\mathrm{r}=-.10, \mathrm{p}<.05)$. Second generation was significantly correlated with age $(\mathrm{r}=-.10, \mathrm{p}<.05)$ and SES $(\mathrm{r}=.11, \mathrm{p}<.05)$. Third generation was significantly correlated with SES $(\mathrm{r}=.16, \mathrm{p}<.05)$, low self-control $(\mathrm{r}=.14, \mathrm{p}<.05)$, violent offending $(\mathrm{r}=.10, \mathrm{p}<.05)$, and the total self-report offending scale $(\mathrm{r}=.08, \mathrm{p}<.05)$. These bivariate findings largely mirror those of previous research wherein first generation immigrants tend to report fewer social and behavioral problems, especially relative to their third generation counterparts.

T-tests were utilized to compare the mean number of offenses between the three specified Hispanic ethnicities (see Table 3). Results suggest a significant difference between the Mexican adolescents and non-Mexicans in self-reported violent offending, such that Mexican adolescents were less likely than non-Mexicans to self-report violent offending $(\mathrm{t}=3.525, \mathrm{p}<.0001)$. There was also a significant difference between Puerto Ricans and non-Puerto Rican subjects relative to self-reported violent offending, such that Puerto Ricans were more likely to self-report violent offending than non-Puerto Ricans $(\mathrm{t}=-2.418, \quad \mathrm{p}<.01)$. There was no significant difference between other Hispanics vs other adolescents (Mexicans and Puerto Ricans) in relation to either self-reported violent or property offending.

Findings also indicated there were no significant differences between the any of the groups with respect to property offending. To summarize, the only differences observed between the Hispanic subgroups were those for violent offending. Mexican adolescents, on average, reported less involvement in violent offending as compared to other subgroups while Puerto Rican adolescents, on average, reported more involvement in violent offending. No differences were observed between any of the groups relative to property offending.

Due to the count nature of the dependent variables, negative binomial regression was utilized to examine the association between the independent variables (ethnicity, generational status, gender, age, SES, delinquent peers, and self-control) and the three dependent variables (property, violent, and overall delinquency). Model 1 regresses property crime on ethnicity and reveals a statistically significant relationship between only age and property offending (coeff. $=.13 ; \mathrm{p}<.001$; see Table 4). By including the demographic controls in the multivariate model, the effects of ethnicity observed in the bivariate analyses are eliminated. Model 2 re-estimates the equation with the inclusion of the generational status (acculturation) measures.

Consistent with the findings from model 1, only age exerted a significant effect on property offending (coeff. $=.13 ; \mathrm{p}<.001)$. The coefficients for the acculturation variables were in the expected direction, though none rose to the level of statistical significance. Model 3 includes the ethnicity and acculturation variables as well as the theoretically relevant controls. The inclusion of these control variables reduces the effect of age on property offending. Surprisingly, only the delinquent peers variable was significantly related to property offending (coeff. $=.07$; $\mathrm{p}<.0001$ ). Contrary to the bulk of existing literature, selfcontrol failed to demonstrate a significant effect on property crime. The insignificance of the ethnicity variables is not surprising given the results from the t-tests.

Table 4. Negative Binomial Models Regressing Property Crime on Independent Variables $(n=737)$

\begin{tabular}{|l|c|c|c|}
\hline & $\begin{array}{c}\text { Model 1 } \\
\text { Coeff. (SE) }\end{array}$ & $\begin{array}{c}\text { Model 2 } \\
\text { Coeff. (SE) }\end{array}$ & $\begin{array}{c}\text { Model 3 } \\
\text { Coeff. (SE) }\end{array}$ \\
\hline \hline$\underline{\text { Variable }}$ & & & \\
Gender (1=Male) & $.19(19)$ & $.20(.19)$ & $.14(.22)$ \\
Age & $.13(.04)^{* * *}$ & $.14(.04)^{* * *}$ & $.01(.05)^{*}$ \\
SES & $.05(.09)$ & $.05(.09)$ & $.01(.10)$ \\
Mexican + & $.01(.33)$ & $.01(.34)$ & $-.19(.37)$ \\
Puerto Rican + & $.23(.38)$ & $.22(.38)$ & $-.23(.43)$ \\
$1^{\text {st }}$ Generation & & $-.10(.46)$ & $-.41(.52)$ \\
$2^{\text {nd }}$ Generation & & $.06(.42)$ & $-.28(.48)$ \\
Delinquent Peers & & & $.07(.01)^{* * *}$ \\
Self-Control & & & $.01(.01)$ \\
\hline
\end{tabular}

Table 5 presents the findings from the second series of regression equations. Model 4 regresses violent crime on the ethnicity variables and demographic controls. Similar to the findings from the previous models predicting property offending, the ethnicity variable failed to exert a significant effect on violent offending. However, sex (coeff. $=.57$; $\mathrm{p}<.01$ ), age (coeff. $=.26 ; \mathrm{p}<.0001$ ), and SES (coeff. $=.16$; $\mathrm{p}<.05$ ) were all significantly related to violent offending. Acculturation was related to violent crime such that first generation youth were significantly less likely to report violence than their third generation counterparts (coeff.=1.15; $\mathrm{p}<.01$ (Model 5). Age (coeff. $=.27 ; \mathrm{p}<.0001$ ) and sex (coeff. $=.57 ; \mathrm{p}<.01)$ remained significant in this model with older students and males more likely to report violent behavior. Model 6 adds the criminological controls to the 
equation. Sex (coeff. $=.49 ; \mathrm{p}<.05)$ and age (coeff. $=.11 ; \mathrm{p}<.05)$ retain their significant effect on violent crime as does generational status (coeff. $=-1.01 ; \mathrm{p}<.05$ ). Similar to the findings from the property crime analyses, delinquent peers exerted a significant, positive effect on self-reported violence (coeff. $=.09 ; \quad \mathrm{p}<.0001)$ while low self-control was insignificant.

Table 5. Negative Binomial Models Regressing Violent Crime on Independent Variables $(n=711)$

\begin{tabular}{|l|c|c|c|}
\hline & $\begin{array}{c}\text { Model 4 } \\
\text { Coeff. (SE) }\end{array}$ & $\begin{array}{c}\text { Model 5 } \\
\text { Coeff. (SE) }\end{array}$ & $\begin{array}{c}\text { Model 6 } \\
\text { Coeff. (SE) }\end{array}$ \\
\hline \hline$\underline{\text { Variable }}$ & & & \\
Gender & $.57(18)^{* *}$ & $.57(.18)^{* *}$ & $.49(.21)^{*}$ \\
$(1=$ Male $)$ & & & \\
Age & $.26(.04)^{* * *}$ & $.27(.04)^{* * *}$ & $.11(.05)^{*}$ \\
SES & $.16(.08)^{*}$ & $.13(.08)$ & $-.10(.10)$ \\
Mexican + & $-.26(.31)$ & $-.28(.30)$ & $-.07(.36)$ \\
Puerto Rican + & $.11(.34)$ & $-.02(.34)$ & $-.11(.41)$ \\
$1^{\text {st }}$ Generation• & & $-1.15(.39)^{* *}$ & $-1.01(.47)^{*}$ \\
$2^{\text {nd }}$ Generation• & & $-.50(.35)$ & $-.53(.42)$ \\
Delinquent Peers & & & $.09(.02)^{* * *}$ \\
Self-Control & & & $.02(.01)$ \\
\hline
\end{tabular}

Table 6 presents the results of models 7-9 which follow the same steps used to estimate the previous six models. Model 7 reveals that sex (coeff. $=.41 ; \mathrm{p}<.01$ ) and age (coeff. $=.21 ; \mathrm{p}<.0001$ ) again significantly predict total overall delinquency while ethnicity fails to impact this outcome. Model 8 is consistent with the findings presented above from the violent crime analyses in that $\operatorname{sex}$ (coeff. $=.43 ; \mathrm{p}<.01$ ), age (coeff. $=.22 ; \mathrm{p}<.0001$ ), and first generation (coeff. $=-.84$; $\mathrm{p}<.001)$ were significantly related to overall offending. Again, first generation immigrants were significantly less likely than third generation immigrants to report involvement in delinquent behavior. Finally, model 9 adds the criminological controls to the equation. In this final model, only sex (coeff. $=.35 ; \mathrm{p}<.05)$, first generation (coeff.=-.95; $\mathrm{p}<.05$ ), and delinquent peers (coeff.=.09; $\mathrm{p}<.0001)$ are significantly related to overall offending. These findings are discussed in greater detail in the following section.

\section{DISCUSSION}

This study examined ethnicity, generational status, and offending among a sample of Hispanic adolescents toward the goal of answering two research questions: 1) Does offending among Hispanic adolescents vary by ethnicity?, and 2) Does acculturation (measured by generational status) impact the likelihood of criminal offending among these same Hispanic adolescents? The findings presented in the current study were largely consistent with the extant literature related to generational status and offending. While previous research has linked acculturation with negative behavioral outcomes, this analysis also indicated that first generation immigrants are significantly less likely to engage in violent delinquency, even after controlling for relevant criminological variables. The study also highlights that Puerto Rican adolescent are involved to a greater extent in violent offending compared to their Mexican and other Hispanic counterparts. Mexican adolescents, on average, reported less violent crime as compared to Puerto Ricans and other Hispanics. No significant differences were observed relative to property offending.

Table 6. Negative Binomial Models Regressing Overall Delinquency on Independent Variables $(n=698)$

\begin{tabular}{|l|c|c|c|}
\hline & $\begin{array}{c}\text { Model 7 } \\
\text { Coeff. (SE) }\end{array}$ & $\begin{array}{c}\text { Model 8 } \\
\text { Coeff. (SE) }\end{array}$ & $\begin{array}{c}\text { Model 9 } \\
\text { Coeff. (SE) }\end{array}$ \\
\hline \hline$\underline{\text { Variable }}$ & & & \\
Gender & $.41(16)^{* *}$ & $.42(.16)^{* *}$ & $.35(.19)^{*}$ \\
$(1=$ Male $)$ & & & \\
Age & $.21(.04)^{* * *}$ & $.22(.04)^{* * *}$ & $.07(.05)$ \\
SES & $.11(.07)$ & $.09(.07)$ & $.05(.09)$ \\
Mexican + & $-.28(.27)$ & $-.30(.27)$ & $-.20(.32)$ \\
Puerto Rican + & $.08(.30)$ & $-.03(.31)$ & $-.17(.36)$ \\
$1^{\text {st }}$ Generation & & $-.84(.35)^{* *}$ & $-.95(.43)^{*}$ \\
$2^{\text {nd }}$ Generation• & $-.41(.31)$ & $-.57(.39)$ \\
Delinquent Peers & & & $.09(.02)^{* * *}$ \\
Self-Control & & & $.01(.01)$ \\
\hline
\end{tabular}

The ethnicity findings above are somewhat consistent with what has been previously reported in the literature with respect to problem behavior among Hispanics. The few studies that have delineated between ethnic subgroups have shown that compared to other Hispanics, Mexicans tend to report less involvement with problem behaviors such as alcohol abuse [27], and the use of marijuana [16,28], inhalants [35], and cocaine [16]. Mexicans are more likely to be from intact families and less likely to grow up in singlefemale headed households (U.S. Census, 2007). Puerto Ricans, on the other hand, are more likely to grow up with divorced or never married parents and in single-female headed households. The tendency for Puerto Ricans to have a greater number of negative outcomes is consistent with previous literature (Amaro et. al, 1990). Puerto Ricans may be more prone to criminal involvement because they tend to reside in greater proximity to native born minorities, therefore lacking insulation by immigrant communities and becoming more vulnerable to delinquency.

The study presented some interesting results that were expected and somewhat consistent with the existing literature. First, age and sex retained significant effects on delinquency, even after adding additional controls to the models. This is consistent with previous literature which links older youth and males to offending, particularly violent offending. Surprisingly, generational status had no significant relationship with property offending, which is 
inconsistent with previous studies. However, generational status was linked with violent and overall offending, with first generation immigrants far less likely to report involvement in delinquent behavior compared to their third generation counterparts. Previous research, with few exceptions [39,51,52], has typically been bivariate in nature, failing to account for other possible causal factors. These studies have suggested that generational status may have a spurious effect on offending and instead work indirectly through other criminological variables. The findings presented here, however, indicate that generational status cannot be explained away by demographics, ethnicity, or criminological constructs such as delinquent peers and low self-control. These findings, then, offer support for the segmented assimilation perspective which argues immigrants will experience increases in negative outcomes across generations - the ill effects of "Americanization".

Perhaps most surprising was the lack of significance of low self-control across the series of regression models. Though the effects of self-control were in the expected directions, none rose to the level of statistical significance. It is unclear as to why this variable, one of the most consistent predictors of delinquency, failed to exert a significant effect on any of the offending variables. Correlations did indicate significant relationships between these measures at the bivariate level though these findings were not replicated at the multivariate level. It is possible that this factor is not as salient for Hispanic adolescents as compared to their white and black counterparts.

\section{LIMITATIONS \& DIRECTIONS FOR FUTURE RESEARCH}

This study is not without limitations. First, because the PHDCN data are comprised of Chicago adolescents, the results cannot be generalized beyond those Hispanics residing within the sampled Chicago neighborhoods. It is possible that results may be different utilizing other datasets. Another limitation may relate to the operationalization of the ethnicity variables. The grouping of Spanish, Central Americans, South Americans, and other Hispanics into a general 'other' category may obscure differences between these subgroups. There were not enough cases within each of the remaining ethnicities to justify their own category and we caution against assuming similarities in behavior across these groups. Finally, generational status was used to measure the level of acculturation of the adolescents. Despite the frequent usage of this variable to measure acculturation, it is not a perfect measure and may not accurately capture the concept. Better measures of acculturation which capture the multidimensionality of the construct may be capable of more accurately parsing out the effects of the acculturation process. For example, the revised Acculturation Rating Scale for Mexican Americans II (ARSMA-II), designed by Cuellar, Arnold, and Maldonado [53], utilizes separate subscales for assessing acculturation processes through a multidimensional approach by measuring cultural orientation toward Mexican and Anglo culture independently. The ARSMA-II is multifactorial and capable of generating multidimensional acculturative types. This type of measure is preferable to linear, unidimensional measures and those that use generational status as a proxy.
There is considerable room for future research in the area of Hispanic crime and victimization. First, the study could be replicated using other samples both with additional waves of the PHDCN and other datasets. Because the PHDCN is a longitudinal data set that offers a wide range of variables, future research using these data may also assess the other predictions of segmented assimilation theory. This theory places some focus on the disadvantaged economy into which "new immigrants" are assimilating. The PHDCN longitudinal data also allows for subjects' unemployment and economic situation to be closely examined over time.

This study can also be replicated using a more accurate measure of acculturation. Generational status has been used as a proxy to measure acculturation, but a more precise operationalization may further clarify these results. As mentioned above, multidimensional measures of acculturation have been developed and may offer greater clarification as to the relationship between acculturation and criminal behavior.

Qualitative research on Hispanic victimization and crime may be another direction for future studies. Examining firsthand the disadvantaged living conditions of ethnic minorities would enable direct observation of the environmental context in which problem behavior occurs. Using qualitative research to study Hispanic victimization and crime would contribute to literature considerably by offering a new, indepth research base from which to further this area of study. Little qualitative research on Hispanics has been conducted since the 1980s and research projects of this kind would open an entirely new area of criminological research. Qualitative research would allow for observation of immigrant communities in their natural settings and provide a means by which subjects are able to communicate their own experiences, in their own words. Furthermore, assimilation may be better measured through qualitative techniques such as ethnography or in-depth interviews which allow respondents to describe their acculturation process for themselves.

Ultimately, the findings produced by this study may raise more questions than it answers. Why are Puerto Rican youth more likely to report violent offending? Why is this not found relative to property offending? Why are Mexicans less likely to report violent delinquency? What is it about being a first generation immigrant that serves as a buffer against adolescent problem behavior? These and other unanswered questions provide a basis for additional research in this vein.

\section{APPENDIX: ITEMS FOR MEASURES}

\section{Criminal Involvement Measures}

\section{Violent Offending}

In the past 12 months have you:

Carried a weapon

Purposely set fire to a house, car, or vacant building

Snatched someone's purse or wallet

Hit someone you live with

Hit someone you did not live with

Attack someone with a weapon

Use a weapon or force to get money or thing from people 
Thrown object like rocks or bottles at people

Shot someone

Shot at someone

Been in a gang fight

Threatened to physically hurt someone

Tried to have sexual relations with someone against their will

\section{Property Offending}

In the past 12 months have you:

Purposely damaged or destroyed property belonging to you

Entered or broken into a building to steal something

Stolen something from a store

Taken something that didn't belong to your from any member of your family

Taken something from a car not belonging to you

Stolen a car or motorcycle

Used credit of bank card without permission

\section{Self-Control Measure}

\section{Inhibitory Control}

Has trouble controlling his/her impulses

Usually can not stand waiting

Can tolerate frustration better than most (reverse code)

Has trouble resisting temptation

Finds self-control easy to learn (reverse code)

\section{Decision Time}

Often says the first thing that comes into his/her head

Likes to plan things way ahead of time (reverse code)

Often acts on the spur of the moment

Always likes to make detailed plans before (s)he does something (reverse code)

\section{Sensation Seeking}

Generally seeks new and exciting experiences and sensations Will try anything once

Sometimes does "crazy" things just to be different

Tends to get bored easily

\section{Persistence}

Generally likes to see things through to the end (reverse code)

Tends to give up easily

Unfinished tasks really bother (reverse code)

Once gets going on something (s)he hates to stop (reverse code)

\section{Delinquent Peers Measure}

In the past year, how many people you spend time with have done the following things:

Skipped school

Gotten in trouble at school

Gotten in trouble at home

Lied, disobeyed, or talked back to adults

Purposely damaged or destroyed property
Stolen something worth $\$ 5$ or less

Stolen Something worth $\$ 5$ but less than $\$ 500$

Stolen something worth more than $\$ 500$

Go into building and steal something

Taken a motor vehicle, car or motorcycle for a ride or drive without the owners permission

Gotten into a physical fight (fist) with schoolmates/coworker or friends

Hit someone with the idea of hurting them

Attacked someone with a weapon with the idea of seriously hurting them

Have used a weapon or force to get money or thing from people

Sold drugs, such as heroin cocaine, crack or LSD (other than marijuana)

Used marijuana or pot

Used any form of alcohol (including wine, liquor, or beer)

Used drugs, such as heroin, cocaine, crack or LSD (other than marijuana)

Used tobacco

How many have had sexual intercourse

\section{ACKNOWLEDGEMENT}

None declared.

\section{CONFLICT OF INTEREST}

None declared.

\section{REFERENCES}

[1] U.S. Census. The nation's Hispanic population-1994 Washington, DC: U.S. Department of Commerce Economics and Statistics Administration 1995. Available online: http:/www.censu s.gov/apsd/www/statbrief/sb95_25e.pdf

[2] U. S. Census. The Hispanic Population: Census 2000 brief Washington, DC: U. S. Department of Commerce Economics and Statistics Administration 2001. Available online: http://www.cens us.gov/prod/2001 pubs/c2kbr01-3.pdf

[3] U.S. Census. Hispanic population passes 40 million. U.S. Census Bureau News. Washington, DC: U. S. Department of Commerce Economics and Statistics Administration 2005. Available online: http://www.census.gov/PressRelease/www/releases/archives/popul ation/005164.html

[4] Grieco EM. Race and Hispanic origin of the foreign-born population in the United States: 2007, American Community Survey Reports, ACS-11. Washington, DC: U.S. Census Bureau 2009

[5] Park R, Burgess E. The city. Chicago: University of Chicago Press 1924

[6] Shaw C, McKay H. Juvenile delinquency and urban areas Chicago: Chicago University Press 1942.

[7] Martinez R, Lee MT. On immigration and crime. Criminal Justice 2000; 1: 485-524.

[8] Martinez R. Latino homicide: immigration, violence, and community. New York: Routledge Press 2002.

[9] Lee MT, Martinez RJ, Rosenfeld R. Does immigration increase homicides? negative evidence from three border cities. Sociol Q 2001; 42: 559-80.

[10] Gordon M. Assimilation in American life: the role of race, religion, and national origins. New York: Oxford University Press 1964.

[11] Portes A, Zhou M. The new second generation: segmented assimilation and its variants. Ann Am Acad Polit Sci 1993; 53: 74 96.

[12] Gans HJ. Second generation decline: scenarios for the economic and ethnic futures of the post-1965 American immigrants. Ethnic Racial Stud 1991; 15: 173-92. 
[13] Zhou M. Segmented assimilation: issues, controversies, and recent research on the new second generation. Int Migr Rev 1997; 31: 975-1008

[14] Morenoff JD, Astor A. Immigration assimilation and crime: Generational differences in youth violence in Chicago. In: Martinez R, Valenzuela, A, Ed. Immigration and crime: race, ethnicity, and violence. New York: New York University Press 2006; pp. 36-63.

[15] Garcia G, Hurwitz, EL, Kraus JF. Acculturation and reported intimate partner violence among Latinas in Los Angeles. J Interpers Violence 2005; 20: 569-79.

[16] Amaro H, Whitaker R, Coffman G, Heeren T. Acculturation and marijuana and cocaine use: findings from HHANES 1982-84. Am J Public Health 1990; 80: 54-60.

[17] Hagan J, Palloni A. Immigration and crime in the United States. In: Smith JP, Edmonston B, Eds. The immigration debate: studies on the economic, demographic, and fiscal effects of immigration. Washington, DC: National Academy Press 1998; pp. 367-87.

[18] Sellin T. Culture and crime: a report of the subcommittee on delinquency of the committee on personality and culture. New York: Social Science Research Council 1938.

[19] Sutherland EH. Principles of criminology. Chicago: Lippencott 1934.

[20] Harris KM. The health status and risk behavior of adolescents in immigrant families. In: Hernandez DJ, Ed. Children of immigrants: health, adjustment, and public assistance. Washington, DC: National Academy Press 1998; pp. 286-347.

[21] U. S. Census. The American Community-Hispanics: 2004. Washington, DC: U.S. Department of Commerce Economics and Statistics Administration 2007. Available online: http://www.censu s.gov/prod/2007pubs/acs-03.pdf

[22] Anderson E. Code of the street. New York: W.W. Norton 1999.

[23] McCord J. Ethnicity, acculturation, and opportunities: a study of two generations. In: Hawkins DF, Ed. Ethnicity, race, and crime. Albany: State University of New York Press 1995.

[24] Aldrich L, Variyam JN. Acculturation erodes the diet quality of U.S. Hispanics. Food Rev 2000; 23: 51-5.

[25] Buriel R, Calzada S, Vasquez R. The relationship of traditional Mexican American culture to adjustment and delinquency among three generations of Mexican American male adolescents. Hispanic J Behav Sci 1982; 4: 41-55.

[26] Burnam A, Hough RL, Karno M, Escobar JI, Telles CA. Acculturation and lifetime prevalence of psychiatric disorders among Mexican Americans in Los Angeles. J Health Soc Behav 1987; 28: 89-102.

[27] Caetano R. Acculturation and drinking patterns among U.S. Hispanics. Br J Addict 1987; 82: 789-99.

[28] Chappin SR, Brook JS. The influence of generational status and psychosocial variables on marijuana use among black and Puerto Rican adolescents. Hispanic J Behav Sci 2001; 23: 22-36

[29] Kaplan MS, Marks G. Adverse effects of acculturation: psychological distress among Mexican American young adults. Soc Sci Med 1990; 31: 1313-9.

[30] Cortes DE. Idioms of distress, acculturation, and depression: the Puerto Rican experience. In: Chun KA, Organista PB, Marin G, Eds. Acculturation: advances in theory, measurement, and applied research. Washington, DC: American Psychological Association 2003; pp. 207-22.

[31] Gong F, Takeuchi DT, Agbayani-Siewert P, Tacata L. Acculturation, psychological distress, and alcohol use: investigating the effects of ethnic identity and religiosity. In: Chun KA, Organista PB, Marin G, Eds. Acculturation: advances in theory, measurement, and applied research. Washington, DC: American Psychological Association 2003; pp. 189-206.

[32] Love AS, Yin Z, Codina E, Zapata JT. Ethnic identity and risky health behaviors in school-age Mexican-American children. Psychol Rep 2006; 98: 735-44.
[33] Zambrana RE, Scrimshaw SCM, Collins N, Dunkel-Schetter C. Prenatal health behaviors and psychological risk factors in pregnant women of Mexican origin: the role of acculturation. Am J Public Health 1997; 87: 1022-6.

[34] Grant BF, Stinson FS, Hasin DS, Dawson DA, Chou SP, Anderson $\mathrm{K}$. Immigration and lifetime prevalence of DSM-IV psychiatric disorders among Mexican Americans and non-Hispanic whites in the United States. Arch Gen Psychiat 2004; 6: 1226-33.

[35] Barrett ME, Joe GW, Simpson DD. Acculturation influences on inhalant use. Hispanic J Behav Sci 1991; 13: 276-96.

[36] De La Rosa M. Prevalence and consequences of alcohol, cigarette, and drug use among Hispanics. Alcohol Treat Q 1998; 16: 21-54.

[37] De La Rosa M. 2002 Acculturation and Latino adolescents' substance use: a research agenda for the future. Substance Use and Misuse 2002; 37: 429-56.

[38] Gilbert MJ. Alcohol consumption patterns in immigrant and later generation Mexican American women. Hispanic J Behav Sci 1987; 9: 299-313.

[39] Neff JA, Hoppe SK, Perea P. Acculturation and alcohol use: drinking patterns and problems among Anglo and Mexican American male drinkers. Hispanic J Behav Sci 1987; 9: 151-81.

[40] Hirschman C. The educational enrollment of immigrant youth: A test of the segmented-assimilation hypothesis. Demography 2001; 38: 317-36

[41] Maldonado-Molina M, Reingle J, Jennings WG, Prado G. Drinking and driving among immigrant and US-born Hispanic young adults: results from a longitudinal and nationally representative study. Addict Behav 2011; 36: 381-8.

[42] Maldonado-Molina M, Reingle J, Tobler A, Jennings WG, Komro KA. Trajectories of physical aggression among Hispanic urban adolescents and young adults: an application of latent trajectory modeling from ages 12 to 18 . Am J Criminal Just 2010; 35: 121-33.

[43] Marz K, Stamatel J. Project on Human Development in Chicago Neighborhoods (PHDCN) data now available. ICPSR Bulletin 2005; 26: 13-7.

[44] Molnar BE, Buka SL, Brennan RT, Holton JK, Earls F. A multilevel study of neighborhoods and parent-to-child physical aggression: results from the Project on Human Development in Chicago Neighborhoods. Child Maltreat 2005; 8: 84-97.

[45] Sampson RJ, Raudenbush SW, Earls F. Neighborhoods and violent crime: a multilevel study of collective efficacy. Science $1997 ; 277$ : 918-24.

[46] Sampson RJ, Morenoff J, Earls F. Beyond social capital: spatial dynamics of collective efficacy for children. Am Sociol Rev 1999; 64: 633-60.

[47] Huizinga D, Esbenson FA, Weihar J. Are there multiple paths to delinquency? Journal of Criminal Law and Criminology 1991; 82: 83-118

[48] Pratt TC, Cullen FT. The empirical status of Gottfredson and Hirschi's general theory of crime: a meta-analysis. Criminology 2000; 38: 931-64.

[49] Gibson C, Sullivan C, Jones S, Piquero A. Does it take a village?: assessing neighborhood effects on children's self-control. J Res Crime Delinquency 2010; 47: 31-62.

[50] Akers RL Social learning and social structure: a general theory of crime and deviance. Boston: Northeastern University Press 1998.

[51] Miller HV, Jennings WG, Alvarez-Rivera L, Lanza-Kaduce L. Self-control, attachment, and deviance among Hispanic adolescents. J Crim Just 2009; 37: 77-84.

[52] Miller HV, Barnes, JC, Hartley R. Reconsidering Hispanic gang membership and acculturation in a multivariate framework. Crime Delinquency 2011; 57: 331-5.

[53] Cuéllar I, Arnold B, Maldonado R. Acculturation rating scale for Mexican Americans-II: a revision of the original ARSMA scale. Hispanic J Behav Sci 1995; 17: 275-304. 